\title{
Pais, Filhos e Deficiência: Estudos Sobre as Relações Familiares
}

Parents, children and disability: Studies on family relations
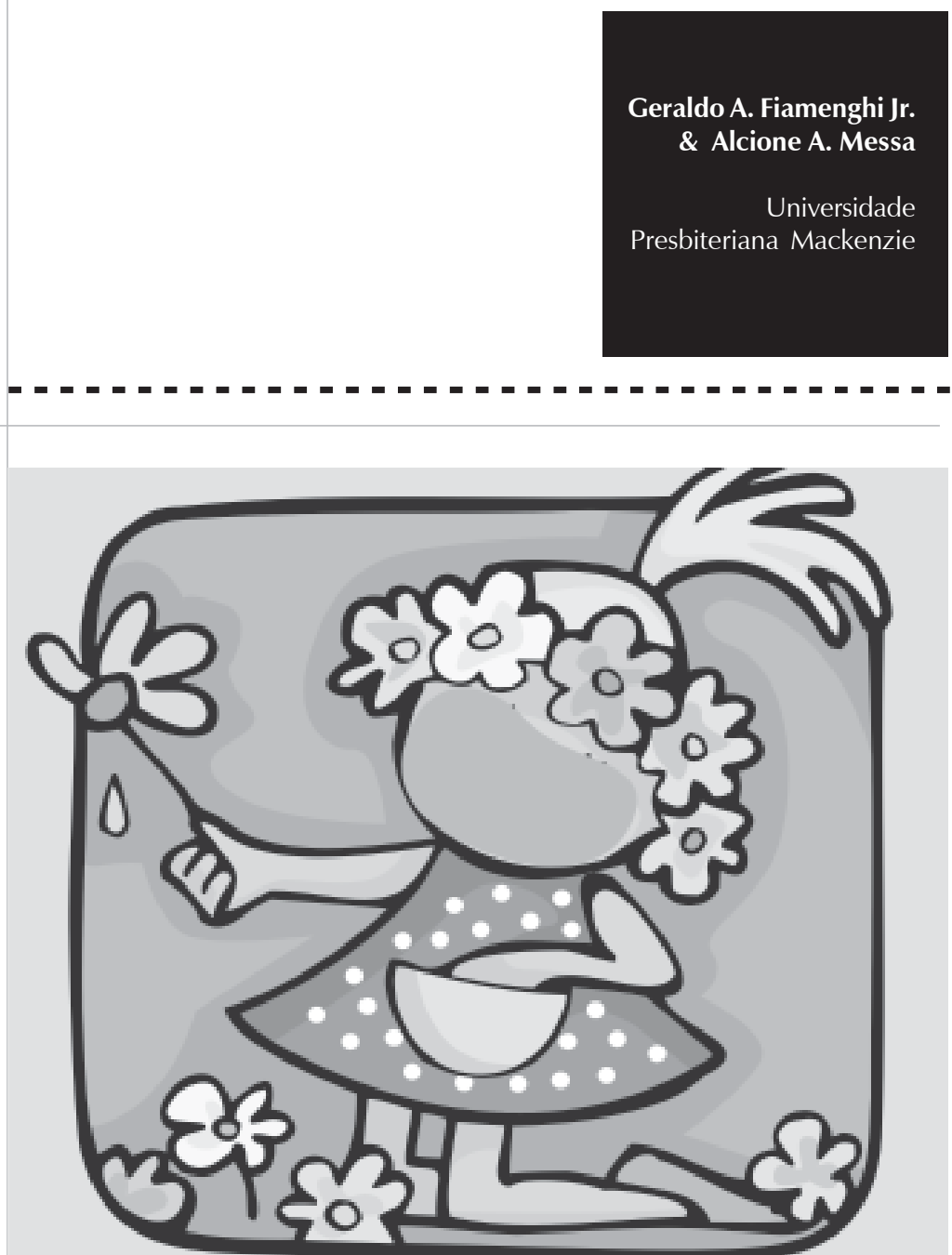


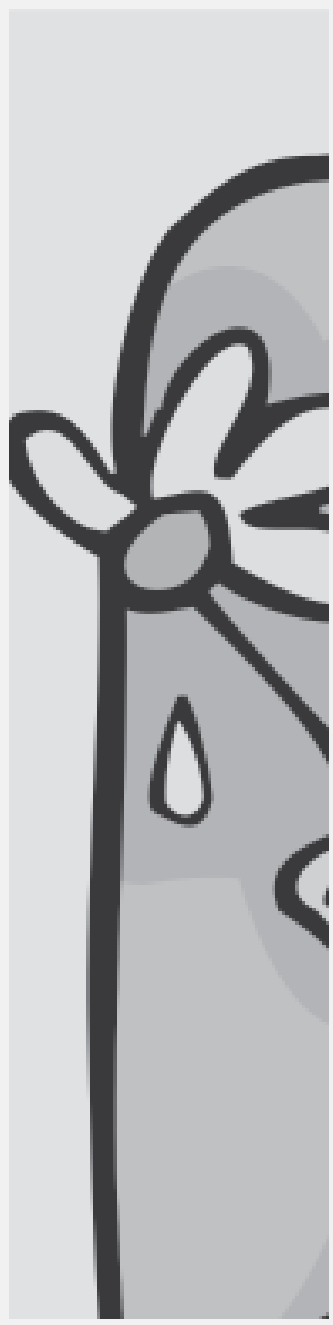

Resumo: Este artigo discute estudos sobre a família atual e as situações que ocorrem quando existe uma criança deficiente na família. Parte do princípio de que não se pode negar a importância de aspectos ambientais no desenvolvimento e de que as famílias são fundamentais para o desenvolvimento das crianças. Quando existe uma criança deficiente na família, esta não irá, necessariamente, causar transtornos familiares, mas a ocorrência destes dependerá de múltiplos fatores, desde as crenças dos pais até os recursos da família em lidar com a deficiência. Além disso, mostra que os pais desejam e merecem ser tratados de forma sensível pelos profissionais que atendem seus filhos.

Palavras-chave: família, relações familiares, crianças portadoras de deficiência, deficiências do desenvolvimento, irmãos.

Abstract:This paper discusses studies on families and situations that may happen when a disabled child is born. It is based on the assertion that it is not possible to deny the importance of environmental aspects on development, and that families are fundamental for the child's development. In the case of the presence of a disabled child in the family, that will not necessarily cause disruption in the family life but that will depend on various factors, varying from parents' beliefs to the resources available to cope with the disability. It also shows that parents wish and deserve to be fairly treated by the professionals who attend their children.

Key words: family, family relations, disabled children, developmental disabilities, siblings.

Em 1998, Harrisescreveu um artigo afirmando que não existe influência dos pais sobre o desenvolvimento das crianças. Essa publicação deu origem a uma nova onda de pesquisas sobre a família, iniciada com uma grande conferência, em 1999, patrocinada pela Robert Wood Johnson Foundation e pelo National Institute of Child Health and Human Development (NICHD), com o objetivo de demonstrar que, de fato, a família exerce fundamental influência sobre o desenvolvimento da criança e os perigos de se considerar aspectos muito específicos quando se trabalha com questões familiares. Desde aquela data, foram publicados vários artigos e livros, na tentativa de esclarecer áreas significativas das relações entre pais e filhos. Inclusive, em relação aos estudos que afirmam serem as diferenças genéticas mais influentes no desenvolvimento do que as familiares (Rowe, 1995), pesquisas demonstram que existem ganhos em relação ao desempenho intelectual nas crianças adotadas (Duyme, Dumaret e Tuomkiewicz, 1999), e que crianças adotadas têm melhor rendimento escolar e melhores condições na vida adulta do que crianças não adotadas que vivem nas mesmas circunstâncias (Maughan, Collishaw e Pickles, 1998).

A família é o primeiro grupo no qual o indivíduo é inserido. Não se pode negar sua importância 
já que, segundo Buscaglia (1997), a família é uma força social que tem influência na determinação do comportamento humano e na formação da personalidade. Pode ser definida como uma unidade social significativa inserida na comunidade imediata e na sociedade mais ampla. É interdependente, ou seja, os relacionamentos estabelecidos entre os familiares influenciam uns aos outros e toda mudança ocorrida nesse sentido irá exercer influência em cada membro individualmente ou no sistema como um todo.

Com o nascimento do primeiro filho, o subsistema parental é formado. As alterações são sentidas pelos pais em diversas esferas do cotidiano e eles devem se organizar para os primeiros cuidados ao bebê. O nascimento de uma criança pode gerar mudanças em toda a estrutura familiar. Segundo Bradt (1995, p.206), "não existe nenhum estágio que provoque mudança mais profunda ou que signifique desafio maior para a família nuclear e ampliada do que a adição de uma criança ao sistema familiar."

"não existe nenhum estágio que provoque mudança mais profunda ou que signifique desafio maior para a familia nucleare ampliada do que a adição de uma criança ao sistema familiar."
A transição para a parentalidade envolve a mudança das identidades individuais de marido e mulher para as de pai e mãe, assim como o relacionamento de casal muda para o de unidade familiar (Delmore-Ko et al., 2000). Em um contexto amplo, quando uma criança nasce, toda a rede de relacionamentos familiares é modificada em seu status, adquirindo uma dialética dos diferentes e novos papéis: os sobrinhos são também primos, os irmãos se tornam tios e sogros e pais se tornam avós (Bradt, 1995).

A parentalidade é uma união de singularidades, em que o casal apresenta ao mundo o produto de sua união. A parentalidade sugere o desempenho de um papel definido e aceito pela sociedade. Os conceitos de pai e mãe são permeados por requisitos prévios, responsabilidades e características, que provocam generalizações de como esse papel deve ser desempenhado (Buscaglia, 1997).

Maccoby (2002) explica que a parentalidade é mais do que uma influência dos pais sobre os filhos, é um conjunto de processos interativos inter-relacionados que têm início com o nascimento da criança. Cada membro entende e dá significado às relações segundo sua história de vida e seus recursos pessoais. Considerando isso, é clara a compreensão de que cada filho desenvolve um tipo de personalidade e vivencia a relação com os pais de acordo com suas expectativas, sentimentos e disponibilidade afetiva.

As influências são recíprocas, ou seja, consistem em uma interação de fatores internos e do ambiente e, portanto, é necessário compreender que o sujeito interage sobre o ambiente e vice-versa. Sendo assim, fica claro que as relações afetam os indivíduos, especialmente dentro do grupo familiar, que é uma formação social que carrega intenso significado.

Kochanska (1997) chegou à conclusão que um estilo parental tem efeitos diferentes sobre os filhos, dependendo do temperamento da criança. Uma tendência atual das pesquisas sobre efeitos dos pais sobre filhos é o estudo de efeitos não compartilhados do desenvolvimento (nonshared effects), demonstrando que as atitudes dos pais têm a tendência de tornar as crianças mais diferentes do que iguais, numa mesma família (Turkheimer e Waldron, 2000). Isso significa que o ambiente tem efeitos sobre as crianças, embora cada criança possa perceber esses efeitos dependendo de suas características particulares.

Ramey (2002) comenta que as propostas radicais sobre mudanças na criação de filhos, que aparecem de tempos em tempos na mídia, sendo tomadas como as formas mais adequadas e atualizadas de se lidar com as 
crianças, servem apenas para confundir pais e legisladores, porque se baseiam em premissas falsas, apesar de sua aparente cientificidade. Muitos são os motivos que levam um casal a planejar o nascimento de um filho ou a se organizar para recebê-lo. Mesmo quando a criança não é planejada, alguns fatores irão determinar o lugar que ela ocupará na família. A decisão de ter um filho está relacionada com o momento de vida dos genitores. O filho pode ser planejado de acordo com o cotidiano dos pais, porém algumas crianças podem ser geradas como substitutas de realizações não alcançadas pelos pais ou quando eles têm pouco contato social ou poucas atividades extradomésticas. Podem ser geradas também para tentar encobrir um momento de crise, falta de intimidade do casal ou como resolução de conflitos.

A influência das relações familiares é clara em famílias com filhos deficientes, pois se trata de uma experiência inesperada, de mudança de planos e expectativas dos pais. Mesmo assim, quando se consideram as famílias cujas crianças nascem com alguma deficiência, as pesquisas atuais também mostram diferenças em relação aos conceitos disseminados socialmente.

Os pais projetam uma criança em suas mentes e, desde o princípio da gravidez, fantasiam sobre o sexo do bebê, o desempenho na escola, a carreira e a orientação sexual que irão ter. O lugar da criança na família é determinado pelas expectativas que os progenitores têm sobre ela. Para Amiralian (1986), a decisão de ter um filho se relaciona diretamente com a realização de desejos dos pais, das suas necessidades e com as gratificações e recompensas que a criança poderá lhes trazer.

As relações familiares são muito diversificadas, e seu funcionamento muda quando alterações ocorrem em um membro ou no sistema como um todo (Silva; Dessen, 2001). O nascimento de uma criança com deficiência, portanto, confronta toda a expectativa dos pais, e a família é acometida por uma situação inesperada. Os planos de futuro para essa criança são abdicados, e a experiência de parentalidade deve ser ressignificada. Segundo Silva e Dessen (2001), a família passa por um processo de superação até que aceite a criança com deficiência mental e institua um ambiente familiar propício para a inclusão dessa criança. Buscaglia (1997) destaca que, mesmo depois do impacto inicial, a presença de uma criança deficiente exige que o sistema se organize para atender as necessidades excepcionais. Esse processo pode durar dias, meses ou anos e mudar o estilo de vida da família, seus valores e papéis. A flexibilidade com que a família irá lidar com a situação depende das experiências prévias, aprendizado e personalidade dos seus membros.

Devido ao significado que o relacionamento fraterno adquire ao longo da vida, mudanças fundamentais na saúde e comportamento de um irmão irão afetar os outros, e essas mudanças correspondem sistematicamente às características da criança, da família, da doença ou deficiência (Lobato, Faust e Spirito, 1988). Segundo Casarin (1999), o diagnóstico da Síndrome de Down em um filho desencadeia, na família, um processo semelhante ao luto. Trata-se de um luto pela perda da fantasia do filho perfeito, da criança sadia. Alguns projetos e expectativas são desfeitos por conta da nova realidade, e a aceitação de um filho com deficiência exige uma reorganização dos valores e objetivos da família (Amiralian, 1986). Os planos da família são geralmente postergados com o nascimento de uma criança, e alguns sacrifícios do casal são transitórios, mas, quando a criança nasce com deficiência, esse adiamento pode se prolongar (Núñez, 2003).

Hanson (2003) estudou famílias cujos filhos com síndrome de Down participaram de um programa de intervenção na infância, o qual
Segundo Casarin (1999), 0 diagnóstico da Síndrome de Down em um filho desencadeia, na família, um processo semelhante ao luto. Trata-se de um luto pela perda da fantasia do filho perfeito, da criança sadia. 
foi reavaliado após vinte e cinco anos. Seus resultados mostram que os pais percebem as características positivas da criança com Síndrome de Down, que variam desde a criança ser considerada como bênção, e passa pelas experiências prazerosas dos pais em observar as aquisições dos filhos e pela facilidade em educar uma criança com Síndrome de Down, quando comparada com os filhos saudáveis, que apresentavam problemas como uso de drogas e gravidez na adolescência, todos ausentes nos adolescentes com Síndrome de Down. Logicamente, esses pais também expressaram sentimentos negativos, como a preocupação com as complicações médicas, o ostracismo e a falta de apoio para as crianças, quando crescem, além de uma rede social muito restrita, quando os filhos atingem a adolescência.

Lobato, Faust e Spirito (1988) analisaram os estudos realizados sobre o relacionamento de irmãos com um indivíduo deficiente, os métodos e questionamentos utilizados nesses estudos e apontaram variáveis fundamentais para a análise dos dados encontrados, entre elas, que as características e as conseqüências impostas pela doença ou deficiência interagem com as características e as funções da estrutura familiar. A inter-relação entre o indivíduo, família e comunidade é importante para estudar a vida dos irmãos, e muitos dos efeitos da doença ou deficiência nos irmãos são mais indiretos ou secundários do que primários, e, mais genericamente, refletem os sistemas de relacionamento.

Cate e Loots (2000) expõem os aspectos positivos, além dos aspectos negativos mencionados pelos irmãos de crianças deficientes. Como aspectos positivos, os irmãos demonstram aumento na maturidade, responsabilidade, altruísmo, tolerância, preocupações humanitárias, senso de proximidade na família, autoconfiança e independência. No entanto, parece que os irmãos se sentem melhor quando a família é mais extensa, as circunstâncias socioeconômicas são melhores, os pais têm uma atitude mais positiva em relação à criança deficiente, os irmãos são mais novos do que a criança deficiente, a criança deficiente ainda é nova e a deficiência é menos grave.

Marciano e Scheuer (2005) descrevem que irmãos de crianças autistas apresentam maior comprometimento em sua qualidade de vida quando comparados com irmãos de crianças com problemas de fala.

Concordando com o fato de que o lado negativo dessa vivência é mais ressaltado do que o positivo, Leonard (1991) ressalta que a maioria das pesquisas sobre o relacionamento de irmãos de crianças com deficiência e/ou doenças crônicas se referem à vulnerabilidade da relação de irmãos de crianças cronicamente enfermas, e não à resiliência. No entanto, vários fatores irão garantir maior resiliência à doença crônica na família, especialmente a idade e o sexo, tanto dos irmãos quanto da criança cronicamente doente, além do tamanho da família e da renda familiar.

A convivência com um irmão deficiente é uma experiência conflitiva (Dodd, 2004), permeada de sentimentos positivos e negativos, que, segundo Silva (1996), deveria ser acompanhada por um psicólogo, não somente para fornecer informações aos irmãos, mas também para orientá-los e compreendê-los em sua relação com um indivíduo deficiente. $\mathrm{O}$ atendimento deve ser contínuo, para trabalhar os conteúdos psíquicos dos irmãos em cada etapa da vida. Nixon e Cummings (1999) realizaram um estudo comparativo de irmãos de crianças deficientes e irmãos de crianças não deficientes para analisar a reação dessas crianças ao stress diário de conflitos relacionados à família. Os resultados revelaram que crianças com irmãos deficientes apresentam maior preocupação com conflitos familiares e experienciam mais afetos negativos em resposta a esses conflitos. Essas crianças assumem mais responsabilidade, esperam maior envolvimento e percebem mais 
ameaça em resposta a todos os tipos de conflitos familiares. Demonstraram também mais problemas de ajustamento.

Segundo os autores, o stress é parte natural de qualquer ambiente familiar, e os irmãos de deficientes podem estar expostos a demandas excessivas que se propagam por outros setores de suas vidas. $\mathrm{O}$ estudo mostrou que essas crianças aprenderam a ficar atentas a problemas na dinâmica familiar e são inclinadas a agir em relação a esses conflitos. A caracterização de tais vivências como experiências de vulnerabilidade ou resiliência dependerá de fatores internos e externos pertinentes ao desenvolvimento da criança. Apesar da variação de reações aos desafios familiares apresentadas pelos indivíduos, os resultados apontam o significado potencial de irmãos e a influência ao funcionamento de outras crianças na família. Outro resultado apresentado se relaciona à qualidade do relacionamento marital em que o grupo de crianças com irmãos deficientes não apresentou mais histórias de conflito conjugal do que o grupo de irmãos de crianças não deficientes.

Esses fatores diferenciam o impacto que a deficiência tem na família e funcionam como atenuantes e protetores, e, em conseqüência, torna diferentes os significados das experiências. Fleitas (2000) também se refere aos fatores de proteção nas relações familiares de crianças com deficiência e propõe, inclusive, um programa de intervenção para pais e irmãos, com foco no apoio, informação, equilíbrio e sensibilidade às necessidades e recursos.

Pelchat et al. (1999) analisaram a eficácia de um programa de intervenção precoce em famílias com um filho deficiente e constataram que esse tipo de programa pode auxiliar significantemente a adaptação dos pais nos primeiros dezoito meses da vida da criança. Em comparação com o grupo controle de pais que não haviam participado de um grupo de intervenção precoce, os pais do programa apresentavam melhor adaptação à deficiência da criança, sentiam-se menos ameaçados por sua situação parental e experienciavam menos stress emocional, ansiedade e depressão.

A participação em grupos de intervenção e programas de auxílio pode ser de muita ajuda aos pais de deficientes, pois auxiliam no incremento de informações e dos recursos de enfrentamento e adaptação, gerando a possibilidade de compartilhar suas vivências com outras pessoas que convivem com uma realidade parecida. Se houver dificuldades para lidar com o nascimento de um filho deficiente, os pais devem procurar o auxílio que julgarem necessário para a adaptação à rotina da deficiência, visando à melhor qualidade dos vínculos familiares.

Em um estudo realizado por Naylor e Prescott (2004) sobre a necessidade de grupos de apoio para irmãos de crianças deficientes, os irmãos sentiram que o grupo os auxiliou no aumento de auto-estima e na tomada de consciência da necessidade de ter mais paciência com o irmão deficiente. Além disso, os participantes experimentaram sentimentos positivos por conhecerem outras crianças que vivenciam a mesma condição de possuírem irmãos com deficiência. O tempo que essas crianças tiveram longe de seus irmãos com deficiência foi considerado importante, por prover mais privacidade e convivência com outras pessoas. A forma como cada família supera uma crise depende de seus recursos e da intensidade do evento. Trata-se de um momento em que coexistem a possibilidade de crescimento, o fortalecimento e a maturidade e o risco de transtornos psíquicos, tanto dos indivíduos como dos vínculos. Alguns fatores irão determinar o significado da experiência em cada família: a história de cada membro, as situações de conflito atravessadas anteriormente e como foram solucionadas, o sistema de crenças, a capacidade de enfrentar situações de mudança, a existência ou não de
A forma como cada família supera uma crise depende de seus recursos e da intensidade do evento. Trata-se de um momento em que coexistem a possibilidade de crescimento, o fortalecimento e a maturidade e $\mathrm{o}$ risco de transtornos psíquicos, tanto dos indivíduos como dos vínculos. 
um lugar para a criança com deficiência dentro do sistema familiar, o nível de expectativas, a capacidade de comunicação do grupo familiar, o nível cultural e socioeconômico da família, a existência ou não da rede de apoio, a capacidade da família de se relacionar com os outros e de buscar ajuda e a possibilidade de que a família esteja enfrentando outra crise simultânea à do nascimento da criança com deficiência (Núñez, 2003).

A idéia de que essas famílias sejam necessariamente abaladas em sua qualidade de vida deve ser revista. Núñez (2003), pesquisando as famílias com filhos deficientes, descreve os conflitos presentes nos vínculos e os indicadores de risco nessas famílias. Conclui que os conflitos familiares não surgem em resultado direto da deficiência, mas em função das possibilidades de a família adaptarse ou não a essa situação.

Assim, a visão do senso comum de que uma criança deficiente irá, necessariamente, produzir conflitos na família não tem comprovação em pesquisas. Famílias com crianças deficientes são uma população de risco, mas isso não quer dizer que esse risco irá concretizar-se.

Corroborando esse aspecto, a pesquisa de Valério (2004) com mães de crianças com deficiência mental e múltipla, em processo de reabilitação, revelou que as mães da amostra apresentaram índices de qualidade de vida satisfatórios. A autora utilizou um instrumento de avaliação genérico, que combina o grau de importância ao grau de satisfação com os aspectos da vida abordados pelo IQV (Índice de Qualidade de Vida). As mães da amostra, portanto, apresentaram índices de qualidade de vida satisfatórios, pois, segundo o IQV, estavam satisfeitas quanto aos aspectos da vida que consideram importantes. Van Riper (2003) ressalta que, mesmo em períodos cheios de incerteza, como o momento do diagnóstico e o período após o nascimento da criança com síndrome de Down, a maioria das famílias desenvolve um funcionamento conjugal e familiar saudáveis. Em seus estudos, a autora obteve relatos de melhora nas relações dos familiares.

Gomes e Bosa (2004), em um estudo comparando grupos de irmãos com transtornos de desenvolvimento e irmãos de crianças com desenvolvimento típico, constataram que não existem diferenças entre os grupos quanto aos indicadores de stress. Segundo as autoras, o stress não deve ser entendido em uma relação linear e causal, na qual um evento determinado é um fator preditor de stress. É necessário adotar uma perspectiva transacional, em que outros fatores são considerados. Sendo assim, a presença de um irmão com transtorno global de desenvolvimento não indica, necessariamente, um estressor para os irmãos. Nesse estudo, os resultados mostram que a ausência de indicadores de stress pode estar relacionada à qualidade das relações familiares e à comunicação que a família desenvolve e a uma rede de apoio satisfatória, além de características individuais, estratégias de enfrentamento e desafios específicos do transtorno.

A literatura destaca que as famílias de indivíduos com deficiência sofrem um impacto e são sensíveis a mudanças de planos. A situação dessas famílias é permeada de sentimentos ambíguos; os recursos de enfrentamento de cada uma em lidar com a deficiência irão determinar o significado da experiência e de todas as vivências dos familiares.

Duvall (1957) propôs uma série de oito estágios para o desenvolvimento familiar, que podem ser assim resumidos: casais recém-casados, nascimento dos filhos, famílias com filhos em idade pré-escolar, famílias com filhos em idade escolar, famílias com filhos adolescentes, filhos que estão deixando a casa, pais de meia idade e familiares idosos. Apesar de ter sido escrito 
há quase cinqüenta anos, seu livro ainda é referência para os profissionais que trabalham com famílias, e os estágios descritos por Duvall podem ser adaptados para o estudo de famílias com crianças deficientes, pois explicam as fases de transição que as famílias vivem no desenvolvimento dos filhos.

No caso das famílias com um indivíduo deficiente, as fases do desenvolvimento podem sofrer influências do processo de adaptação da família também à deficiência. Além das crises de transição naturais do grupo familiar, a família lida com as peculiaridades da deficiência, suas características e evolução. Os pais com filhos deficientes vivem preocupações durante toda a vida, do nascimento do bebê até a velhice, principalmente pela inexistência de instituições que possam cuidar das pessoas deficientes à medida que elas envelhecem.

Nos últimos anos, essa preocupação com o envelhecimento tem se agravado, porque, com o avanço de técnicas cirúrgicas e de tratamentos especializados, a expectativa de vida de pessoas deficientes aumentou significativamente.

Miltiades e Pruchno (2001) realizaram uma pesquisa com mães de filhos deficientes adultos e concluíram que elas ainda continuam vivendo situações de cuidado e de responsabilidades pela vida dos filhos, negociando com as agências formais de apoio ou auxiliando nas atividades da vida diária, ou seja, assumindo um papel vitalício de cuidadoras.

A rede de apoio e os serviços disponibilizados para tratamento e acompanhamento da pessoa com deficiência parecem favorecer a adaptação da família, que necessita ser atendida com recursos e profissionais adequados. Nos últimos anos, também podemos perceber uma preocupação cada vez maior com a forma pela qual os profissionais de saúde devem relacionar-se com os familiares de crianças deficientes.
Um dos aspectos mais discutidos pelos pais em relação aos profissionais da saúde diz respeito à forma pela qual eles recebem a notícia da deficiência dos filhos. A maioria dos pais relata um sentimento de descaso e falta de sensibilidade dos profissionais, quando anunciam a deficiência do bebê recém-nascido aos pais (Marchese, 2002).

Outro aspecto que se observa nos pais é a precariedade de informações sobre as deficiências dos filhos (Fiumi, 2003). A maioria dos pais não têm acesso a informações e, em conseqüência, muitas vezes não conseguem seguir as orientações dos profissionais.

Os pais se sentem despreparados para lidar com o evento da deficiência. A busca por informações sobre as peculiaridades do quadro clínico e os cuidados inerentes à condição se fazem necessários, e eles precisam entender e aprender a lidar com um novo corpo de conhecimento relacionado ao diagnóstico e ao sistema de apoio disponível.

A diminuição da ansiedade dos pais acontece com o aumento do conhecimento que adquirem sobre a deficiência, a doença ou a condição crônica. Esse conhecimento é gerado pela intensidade e pela continuidade dos cuidados (Furtado; Lima, 2003). Os pais necessitam de orientação para a disciplina e a educação dessas crianças (Buscaglia, 1997), e as dificuldades podem surgir devido à idéia de que já são crianças limitadas e restritas em muitos aspectos. Os pais não as educam e treinam como os outros filhos, porém, mesmo dentro das limitações, essas crianças precisam de conceitos e limites básicos para a convivência em família e sociedade.

Concluindo, o psicólogo que atua com famílias em que haja algum membro deficiente deve ter em mente, em primeiro lugar, que a presença da família é fundamental para o desenvolvimento da criança. A partir do momento em que nascemos, já nos encontramos inseridos nessa estrutura social
Concluindo, o psicólogo que atua com familias em que haja algum membro deficiente deve ter em mente, em primeiro lugar, que a presença da familia é fundamental para o desenvolvimento da criança. 
básica, em que interações primárias são estabelecidas para garantir nossa sobrevivência. Além disso, apesar de os aspectos genéticos serem importantes, o meio exerce fator relevante na determinação daas características comportamentais da criança. Propostas 'alternativas' para a criação de filhos devem ser consideradas com muita cautela, porque não há evidências de sua efetividade.

Outro ponto fundamental para o psicólogo é perceber que as famílias cujas crianças são deficientes não serão, necessariamente, problemáticas, desde que algumas condições sejam consideradas, entre elas o apoio familiar, econômico e social. Ter um irmão deficiente não significa ter problemas, pois as relações entre irmãos dependerão da estrutura familiar, das crenças da família sobre deficiência, da ordem de nascimento, da idade das crianças e do nível socioeconômico da família. Tratase de uma vivência que compreende aspectos negativos e também ganhos. O risco da ocorrência de distúrbios coexiste com a possibilidade de crescimento, fortalecimento e maturidade dos indivíduos e seus vínculos. Com referência à orientação e psicoterapia, o psicólogo precisa perceber que muitas famílias cujas crianças são deficientes não conhecem os aspectos técnicos referentes à deficiência, e, por isso, precisam ser tratadas de forma sensível e respeitosa pelos profissionais. As informações sobre as características da deficiência podem também abranger dados sobre os serviços disponíveis para tratamento e apoio dessas famílias. A postura profissional sensibilizada para as necessidades diretas das famílias de deficientes precisa estar aliada à propagação de uma idéia mais positiva e realista da deficiência.

Os profissionais, portanto, precisam compreender os pais como parceiros ativos e significativos ao trabalhar com crianças, deficientes ou não. Os pais conhecem os filhos muito profundamente, e os psicólogos necessitam tanto respeitar e utilizar esse conhecimento como auxiliar no processo terapêutico.

Geraldo A. Fiamenghi Jr.

Pós-Graduação em Distúrbios do Desenvolvimento da Universidade Presbiteriana Mackenzie /

Faculdade de Psicologia da Pontifícia Universidade Católica de Campinas.

Alcione A. Messa -

Universidade Presbiteriana Mackenzie

R. da Consolação, 896, 60 andar, s. 62

CEP.:01302-907 São Paulo, SP.

tel: (11) 32368707 fax: (11) 32368600

E-mail: fiamenghi@mackenzie.br 
AMIRALIAN, M.L.T.M. Psicologia do Excepcional. São Paulo: EPU, 1986.

BRADT, J.O. Tornando-se Pais: Famílias com Filhos Pequenos. In: B. Carter \& M. McGoldrick. As Mudanças no Ciclo de Vida Familiar: uma Estrutura para a Terapia Familiar. Trad. Maria Adriana Veríssimo Veronese. $2^{2}$ ed. Porto Alegre: Artmed, 1995.

BUSCAGLIA, L. Os Deficientes e seus Pais. Trad. Raquel Mendes. 3aㅡ ed. Rio de Janeiro: Record, 1997

CASARIN, S. Aspectos Psicológicos na Síndrome de Down. In: J. S Schwartzman (ed.). Síndrome de Down. São Paulo: Mackenzie, 1999.

CATE, I.M.P-T; LOOTS, G.M.P. Experiences of Siblings of Children with Physical Disabilities: an Empirical Investigation. Disability and Rehabilitation, Ipswich: Taylor \& Francis, 22(9), pp. 399-408, 2000

DELMORE-KO, P.; PANCER, S.M.; HUNSBERGER, B.; PRATT, M Becoming a Parent: Relation between Prenatal Expectations and Postnatal Experience. Journal of Family Psychology, Washington, DC: APA, v. 14, n. 4, pp. 625-640, 2000

DUVALL, E. Family Development. Filadélfia: Lippincott, 1957

DUYME, M.; DUMARET, A.; TUOMKIEWICZ, S. How Can we Boos IQ's of "Dull Children": a Late Adoption Study. Proceedings of the National Academy of Science, Palo Alto, CA: National Academy of Sciences.96(15), pp. 8790-8794, 1999.

FIUMI, A. Orientação Familiar: o Profissional Fisioterapeuta segundo a Percepção das Mães de Crianças Portadoras de Paralisia Cerebral. Dissertação de Mestrado em Distúrbios do Desenvolvimento. Universidade Presbiteriana Mackenzie, São Paulo, 2003

FLEITAS, J. When Jack Fell down...jill Came Tumbling after: Siblings in the Web of Illness and Disability. MCN., Hagerstown, MD: Lippincott Williams \& Wilkins, Inc.25(5), pp. 267-273, 2000

FURTADO, M.C.C.; LIMA, R.A.G. O Cotidiano da Família com Filhos Portadores de Fibrose Cística: Subsídios para a Enfermagem Pediátrica. Revista Latino-Americana de Enfermagem, Ribeirão Preto: Escola de Enfermagem da USP.v. 11, n. 1, pp. 66-73, jan./fev., 2003

GOMES, V.F;; BOSA, C. Estresse e Relações Familiares na Perspectiva de Irmãos de Indivíduos com Transtornos Globais do Desenvolvimento. Estudos de Psicologia, Natal, RN: UFRN v. 9, n. 3, pp.553-561, 2004.

HANSON, M. Twenty-five Years after Early Intervention. A Followup of Children with Down Syndrome and their Families. Infants and Young Children, Washington, DC: Lippincott Williams \& Wilkins, 16(4), pp. 354-365, 2003

HARRIS, JR. The Nurture Assumption. New York: Free Press, 1998 KOCHANSKA, G. Multiple Pathways to Conscience for Children with Different Temperaments: from Toddlerhood to Age 5. Developmental Psychology, Washington, DC: APA.33, pp.228-240, 1997.

LEONARD, B. Siblings of Chronically Ill Children: a Question of Vulnerability Versus Resilience. Pediatric Annals, Chicago, II: The American Medical Association, 20(9), pp. 501-506, 1991.

LOBATO, D.; FAUST. D.; SPIRITO, A. Examining the Effects of Chronic Disease and Disability on Children's Sibling Relationships. Journal of Pediatric Psychology, Oxford: Oxford U. Press. v. 13, n. 3, 1988, pp. 389-407.
MACCOBY, E. Parenting Effects: Issues and Controversies. In: J.G. Borkowski; S.L. Ramey; M. Bristol-Power (ed.). Parenting and the Child's World. New Jersey: Lawrence Erlbaum Associates, Publishers, 2002.

MARCHESE, D.M.A. O Nascimento do Cidadão Diferente: Prognóstico ou Julgamento? Dissertação de Mestrado em Distúrbios do Desenvolvimento. Universidade Presbiteriana Mackenzie, São Paulo, 2002.

MARCIANO, A.R.F; SCHEUER, C.I. Quality of Life in Siblings of Autistic Patients. Revista Brasileira de Psiquiatria, São Paulo: Associação Brasileira de Psiquiatria., 27(1), pp. 67-69, 2005.

MAUGHAN, B.; COLLISHAW, S.; PICKLES, A. School Achievement and Adult Qualifications among Adoptees: a Longitudinal Study. Developmental Psychology, Washington, DC: APA, 39, pp. 669-85, 1998.

MILTIADES, H.B; PRUCHNO, R. Mothers of Adults with Developmental Disability: Change over Time. American Journal of Mental Retardation, 106(6), pp. 548-561, 2001.

NAYLOR, A.; PRESCOTT, P. Invisible Children? The Need for Support Groups for Siblings of Disabled Children. British Journal of Special Education, v. 31, n. 4, pp. 199-206, 2004.

NIXON, C.L.; CUMMINGS, E.M. Siblings Disability and Children's Reactivity to Conflicts Involving Family Members. American Psychological Association, v. 13, n. 2, pp. 274-285, 1999.

NúÑ̃EZ, B. La Familia con un Hijo con Discapacidad: sus Conflictos Vinculares. Archives Argentinian of Pediatry, Buenos Aires: Sociedad Argentina de Pediatria, 101(2), pp. 133-42, 2003.

RAMEY, SL. The science and art of parenting. In: J.G. BORKOWSKI S.L. RAMEY; M. BRISTOL- PELCHAT, D.; BISSON, J.; RICARD, N.; PERREAULT, M.; BOUCHARD, J.-M. Longitudinal Effects of an Early Family Intervention Programme on the Adaptation of Parents of Children with a Disability. International Journal of Nursing Studies, Londres: Elsevier, v. 36, pp. 465-477, 1999.

BORKOWSKI, J. G.; RAMEY, S. L.; BRISTOL-POWER, M. (ed.) Parenting and the Child's World. New Jersey: Lawrence Erlbaum Associates, Publishers, 2002.

ROWE, D.C. The Limits of Family Influence: Genes, Experience and Behavior. New York: Guilford Press, 1995.

SILVA, N.L.P.; DESSEN, M.A. Deficiência Mental e Família: Implicações para o Desenvolvimento da Criança. Psicologia: Teoria e Pesquisa, v. 17, n. 2, pp. 133-141, 2001.

TURKHEIMER, E.; WALDRON, M. Nonshared Environment: a Theoretical Methodological and Quantitative Review. Psychological Bulletin, 126, pp. 78-108, 2000.

VALÉRIO, N. Qualidade de Vida de Mães de Crianças Deficientes. Dissertação de Mestrado em Distúrbios do Desenvolvimento. Universidade Presbiteriana Mackenzie, São Paulo, 2004.

VAN RIPER, M. A Change of Plans. American Journal of Nursing, $v$. 103, n. 6, pp. 71-74, Jun., 2003.
Referências 